\title{
Certain Types of Groupoids
}

\section{Taghreed Hur Majeed ${ }^{1} \quad$ Deyaa Hussain Ali $^{2}$}

\section{Mathematics Department Education College University of Al-Mustansirah}

$\underline{\text { taghreedmajeed@uomustansiriyah.edu.iq }^{1} \quad \text { taghreedmajeed@yahoo.com }^{1} \quad \text { deyaa_h_ali@yahoo.com }}{ }^{2}$

Recived : 5\2\2019

Revised : $14 \backslash 2 \backslash 2019$

Accepted : 17\2\2019

Available online : $22 / 4 / 2019$

\begin{abstract}
:
In this work, we try to construct anew types of groupoids and disucss their properties.
\end{abstract}

Keywords: Groupoid, Descarts groupoid, Direct sum of two groupoid, Tensor product of two groupoid, Action groupoid. Classifiacation subject classification: $425 \mathrm{~F}$ 


\section{Introduction:}

The conceept of groupoid is one of the means by which the twentieth century reclaims the original domain of appliication of group concept. In 1920's Brandt and Baer gave the algebraic theory of groupoid .In 1950's Ehresmann introduced the groupoid in to the differential geometry. Also the concept of groupoid action is due originally to "Ehreesmann (1959), generalizing the group action in his work on fibre spaces". Dieudonne, J.; introduce to modern analysis to Lie groupoid (1968). Berson, G.E.; introduce to compact groupoid (1972). Al-Taai,A.A. (1988), gave the action of groupoid. Al-Taai, A. A. and Bedaiwi (1999) , gave representation of topological groupoid. Mahdi J.H.; (2006) gave to vector groupoid and isometries. Taghreed H. M. (2010) gave to some results of Lie group and Lie algebra. In this paper, we study the basic construction of groupoid space and we divide into two sections. In section one, we give definition examples and theorems about groupoid and morphism of groupoid. In section two, we introduce subgroupoid, normal subgroupoid and transitive.

1. Category, Fiber product, Groupoid, morphism of groupoid .

\section{(1.1) Definition(2)}

"A category $\mathrm{C}$ consist of :

(a) A class of objects

(b) For every ordered pair of objects $\mathrm{X}$ and $\mathrm{Y}$ asset hom $(X, Y)$ of morphism with domain $X$ and range $\mathrm{Y}$, if $\mathrm{f} \in \operatorname{hom}(\mathrm{X}, \mathrm{Y})$ we write $\mathrm{f}: \mathrm{X} \rightarrow \mathrm{Y}$.

(c) For every ordered triple of opjects $\mathrm{X}, \mathrm{Y}$, and Z a function associating to a pair of morphism $f$ $: \mathrm{X} \rightarrow \mathrm{Y}$ and

$\mathrm{g}: \mathrm{Y} \rightarrow \mathrm{Z}$ their composite $\mathrm{gf}: \mathrm{X} \rightarrow \mathrm{Z}$.

These satisfy the following two axioms : (i) Associativity : if $\mathrm{f}: \mathrm{X} \rightarrow \mathrm{Y}, \mathrm{g}: \mathrm{Y} \rightarrow \mathrm{Z}$ and $h: Z \rightarrow W$ then $h(g f)=(h g) f$

(ii) Identity :For every object $\mathrm{Y}$ there is a morphism $\mathrm{I}_{\mathrm{Y}}: \mathrm{Y} \rightarrow \mathrm{Y}$ such that if $\mathrm{f}: \mathrm{X} \rightarrow \mathrm{Y}$, then $I_{Y} f=f$ and if $h: Y \rightarrow Z$ then $h I_{Y}=h "$

\section{(1.2) Notation's}

(1) The category of sets and maps which we denote by $\mathrm{M}$.

(2) The category of continuous maps and topological spaces which we denote by $\mathrm{T}$.

\section{(1.3) Definition(5)}

"A pair of sets $(\mathrm{T}, \mathrm{Q})$ is a groupoid on which are given :

(1) Two surjections $\alpha, \beta: T \rightarrow Q$ called the source and the target mapping respectively

(2) An injection $\lambda: T \rightarrow Q$ called the mapping of unities satisfying $\alpha \mathrm{o} \lambda=\mathrm{I}_{\mathrm{Q}}$ and $\beta \mathrm{o} \lambda=\mathrm{I}_{\mathrm{Q}}$ ( where $_{\mathrm{Q}}$ : $\mathrm{Q} \rightarrow \mathrm{Q}$ is the identity mapping on $\mathrm{Q}$ )

(3) A law of partial composition $\gamma$ in $\mathrm{T}$ define as a law of composition

" $\mathrm{T} * \mathrm{~T}=\left\{\left(t, t^{\prime}\right) \in \mathrm{T} \times \mathrm{T} \quad \mid \alpha(t)=\beta\left(t^{\prime}\right)\right\} " \quad$ "fibree product of $\alpha$ and $\beta$ over Q"

such that

(a)'“ $\gamma\left(t, \gamma\left(t_{1}, t_{2}\right)\right)=\gamma\left(\gamma\left(t, t_{1},\right), t_{2}\right)$,for all $\left(t, t_{1}\right),\left(t_{1}, t_{2}\right)$ $\in \mathrm{T} * \mathrm{~T}$ "

(b) " $\alpha\left(\gamma\left(t_{1}, t_{2}\right)\right)=\alpha\left(t_{2}\right), \beta\left(\gamma\left(t_{1}, t_{2}\right)\right)=\beta\left(t_{1}\right)$ for each $\left(t_{1}, t_{2}\right) \in \mathrm{T} * \mathrm{~T} "$

(c) " $\gamma(t, \lambda(\alpha(t)))=t$ and $\gamma(\lambda(\beta(t)), t)=t$,for all $t \in \mathrm{T}$ "

(4) A biijection $\sigma: \mathrm{T} \rightarrow \mathrm{T}$ called the inveersion of $\mathrm{T}$ satisfying

(a) $\alpha(\sigma(t))=\beta(t), \beta(\sigma(t))=\alpha(t)$, for all $t \in \mathrm{T}$

(b) $\gamma(\sigma(t), t)=\lambda(\alpha(t)), \gamma(t, \alpha(t))=\lambda(\beta(t))$, for any $t \in \mathrm{T}$

We write $\sigma(t)=t^{-1}$ called the inverse element of $t \in \mathrm{T}$ and $\lambda(\mathrm{x})=\tilde{\mathrm{x}}$ called the unit element in $\mathrm{T}$ associated to the element $\mathrm{x} \in \mathrm{Q}$. 
Also, we write $\gamma\left(t, t^{\prime}\right)=t t^{\prime}$. $\quad \mathrm{T}$ is called the groupoid and $\mathrm{Q}$ is called the base. Also, we say that $\mathrm{T}$ is a groupoid on Q" .

\section{(1.4) Remark(1)}

"If $(\mathrm{T}, \mathrm{Q})$ be any groupoid then :

(i) The sub set of $T_{-1} ; T_{x}=\alpha(x)$ is called the $\alpha$ fiber at $\mathrm{x} \in \mathrm{Q},{ }_{\mathrm{y}} \mathrm{T}=\beta$ (y) is called the $\beta$-fiber at $\mathrm{y} \in \mathrm{Q}$ and ${ }_{\mathrm{y}} \mathrm{T}_{\mathrm{x}}=\mathrm{T}_{\mathrm{x}} \cap_{\mathrm{y}} \mathrm{T}$ the set

of elements in $\mathrm{T}$ which have $\mathrm{x}$ as a source and $\mathrm{y}$ as a target"

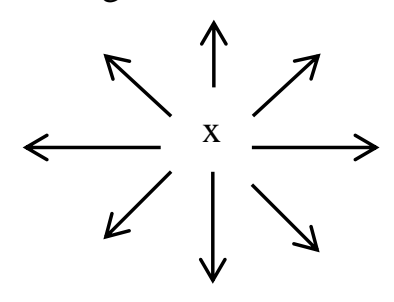

$\alpha$ - fiber at $\mathrm{x}$

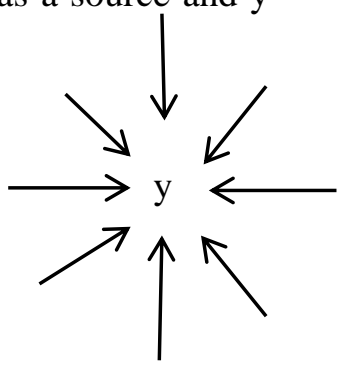

$\beta$ - fiber at $y$ (ii) “ ${ }_{x} T_{x}$ is a group under the restriction $\gamma$ on ${ }_{x} T_{x} \times{ }_{x} T_{x}$ with unity

$\lambda(\mathrm{x})$ called the vertex (isotropy) group at $\mathrm{x} \in \mathrm{Q}$.

(iii) The map $\tau: T \rightarrow Q \times Q ; \tau(t)=(\beta(t), \alpha(t))$ its said to be the transitor of $T$ and ${ }_{y} T_{x}=\tau^{-1}(y, x)$, for all $\mathrm{x}, \mathrm{y} \in \mathrm{Q}$ ".

\section{(1.5) Example}

Let $\mathrm{Q}$ be anon-empty set . The Cartesian product $\mathrm{T}=\mathrm{Q} \times$ é $\times$ é $\times \mathrm{Q}$ is a groupoid of base $\mathrm{Q}$ as follows

$\alpha=\mathrm{pr}_{4}, \beta=\mathrm{pr}_{1}, \lambda(\mathrm{x})=(a$,é,é, $a)$ for all $a \in \mathrm{Q}$ where éxé is the identity element in composition is given by $\left(\mathrm{z}, e ́, e ́, w^{\prime}\right)(\mathrm{w}, e ́, e ́, \mathrm{x})=(\mathrm{z}, \gamma$ (é,é,é,é),x) wherever $w^{\prime}=w$ and $\gamma$ is a law of composition in $\dot{\eta} \times \eta ́$. The invers element of ( $a$,é,é,y) is (y,é,é, $a)$, where $\sigma$ is the inversion law of $\mathrm{T}$. This groupoid is called DeŚcartes groupoid

\section{(1.6) Definition(8)}

"Let $\mathrm{T}_{1}$ and $\mathrm{T}_{2}$ be groupoids the Tensor product of $\mathrm{T}_{1} \otimes \mathrm{T}_{2}$ consist of linear combinations of elements of the form $t_{1} \otimes t_{2}$ where $t_{1} \in \mathrm{T}_{1}$ and $t_{2} \in \mathrm{T}_{2}$ with the following relations :

(i) $\mathrm{c}\left(t_{1} \otimes t_{2}\right)=\left(\mathrm{c} t_{1}\right) \otimes t_{2}=t_{1} \otimes\left(\mathrm{c} t_{2}\right)$, for any scalars c

(ii) $\left(t_{1} \otimes t_{2}\right)+\left(t_{1}^{\prime} \otimes t_{2}^{\prime}\right)=\left(t_{1}+t_{1}^{\prime}\right) \otimes t_{2}$. (iii) $\left(t_{1} \otimes t_{2}\right)+\left(t_{1} \otimes t_{2}^{\prime}\right)=t_{1} \otimes\left(t_{2}+t_{2}^{\prime}\right)$ for all $t_{1}, t_{1}^{\prime} \in \mathrm{T}_{1}$ and $t_{2}, t_{2}^{\prime} \in \mathrm{T}_{2}^{\prime \prime}$.

\section{(1.7) Theorem:}

Let $\left(\mathrm{T}_{1}, \mathrm{Q}_{1}\right)$ and $\left(\mathrm{T}_{2}, \mathrm{Q}_{2}\right)$ are both groupoids then the tensor product $\left(\mathrm{T}_{1} \otimes \mathrm{T}_{2}, \mathrm{Q}_{1} \otimes \mathrm{Q}_{2}\right)$ be a groupoid .

\section{Proof:}

(1) $\alpha, \beta: T_{1} \otimes T_{2} \rightarrow Q_{1} \otimes Q_{2}$

$\alpha\left(v_{1} \otimes v_{2}\right)=w_{1} \otimes w_{2}, \beta\left(v_{1} \otimes v_{2}\right)=w_{1} \otimes w_{2}$, for all $v_{1} \otimes v_{2} \in \mathrm{T}_{1} \otimes \mathrm{T}_{2}$ and $w_{1} \otimes w_{2} \in \mathrm{Q}_{1} \otimes \mathrm{Q}_{2}$,

(2) $\lambda: Q_{1} \otimes Q_{2} \rightarrow \mathrm{T}_{1} \otimes \mathrm{T}_{2}$

$\lambda\left(w_{1} \otimes w_{2}\right)=v_{1} \otimes v_{2}$,

$(\alpha \mathrm{o} \lambda)\left(w_{1} \otimes w_{2}\right)=\alpha\left(\lambda\left(w_{1} \otimes w_{2}\right)\right)=\alpha\left(v_{1} \otimes v_{2}\right)=($ $\left.w_{1} \otimes w_{2}\right)$

$(\beta \circ \lambda)\left(w_{1} \otimes w_{2}\right)=\beta\left(\lambda\left(w_{1} \otimes w_{2}\right)\right)=\beta\left(v_{1} \otimes v_{2}\right)=$ $\left(w_{1} \otimes w_{2}\right)$,

Where

$\alpha \mathrm{o} \lambda=\mathrm{I}_{\mathrm{Q}_{1} \otimes \mathrm{Q}_{2}}, \beta \mathrm{o} \lambda=\mathrm{I}_{\mathrm{Q}_{1} \otimes \mathrm{Q}_{2}}$ and

$\mathrm{I}_{\mathrm{Q}_{1} \otimes \mathrm{Q}_{2}}: \mathrm{Q}_{1} \otimes \mathrm{Q}_{2} \rightarrow \mathrm{Q}_{1} \otimes \mathrm{Q}_{2}$

(3) $\left(\mathrm{T}_{1} \otimes \mathrm{T}_{2}\right) *\left(\mathrm{~T}_{1} \otimes \mathrm{T}_{2}\right)=\left\{\left(v_{1} \otimes v_{2}, v_{1}^{\prime} \otimes v_{2}^{\prime}\right.\right.$

)$\in\left(\mathrm{T}_{1} \otimes \mathrm{T}_{2}\right) \times\left(\mathrm{T}_{1} \otimes \mathrm{T}_{2}\right)$ :

$\left.\alpha\left(v_{1} \otimes v_{2}\right)=\beta\left(v_{1}^{\prime} \otimes v_{2}^{\prime}\right)\right\}$ " fibre product of $\alpha$ and $\beta$ over $Q_{1} \otimes Q_{2}$ "

Such that

(a) $\gamma\left(v \otimes r, \gamma\left(v_{1} \otimes v_{1}, v_{2} \otimes v_{2}\right)\right)$

$=\gamma\left(v \otimes r, v_{2} \otimes v_{2}\right)=v_{2} \otimes v_{2}$

$\gamma\left(\gamma\left(v \otimes r, v_{1} \otimes v_{1}\right), v_{2} \otimes v_{2}\right)=\gamma\left(v_{1} \otimes v_{1}, v_{2} \otimes v_{2}\right)$

$=r_{2} \otimes r_{2}$

So we have $\gamma\left(v \otimes v, \gamma\left(r_{1} \otimes v_{1}, v_{2} \otimes v_{2}\right)\right)=$ $\gamma\left(\gamma\left(v \otimes r, v_{1} \otimes v_{1}\right), v_{2} \otimes v_{2}\right)$

For all $\left(v \otimes v, v_{1} \otimes v_{1}\right)$,

$\left(v_{1} \otimes v_{1}, v_{2} \otimes v_{2}\right) \in\left(\mathrm{T}_{1} \otimes \mathrm{T}_{2}\right) *\left(\mathrm{~T}_{1} \otimes \mathrm{T}_{2}\right)$

(b) $\left.\alpha\left(\gamma_{1} \otimes v_{1}, v_{2} \otimes v_{2}\right)\right)=\alpha\left(v_{2} \otimes v_{2}\right)=w_{2} \otimes w_{2}$

$\alpha\left(v_{2} \otimes v_{2}\right)=w_{2} \otimes w_{2}$

So we get $\alpha\left(\gamma\left(r_{1} \otimes v_{1}, v_{2} \otimes v_{2}\right)\right)=\alpha\left(v_{2} \otimes v_{2}\right)$, $\beta\left(\gamma\left(v_{1} \otimes v_{1}, v_{2} \otimes v_{2}\right)\right)=\beta\left(v_{1} \otimes v_{1}\right)=w_{1} \otimes w_{1}$ $\beta\left(v_{1} \otimes v_{1}\right)=w_{1} \otimes w_{1}$

So we get , $\beta\left(\gamma\left(v_{1} \otimes v_{1}, v_{2} \otimes v_{2}\right)\right)=\beta\left(v_{1} \otimes v_{1}\right)$ for each $\left(v_{1} \otimes v_{1}, v_{2} \otimes v_{2}\right) \in\left(\mathrm{T}_{1} \otimes \mathrm{T}_{2}\right) *\left(\mathrm{~T}_{1} \otimes \mathrm{T}_{2}\right)$ 
(c) $\gamma\left(v_{1} \otimes v_{2}, \lambda\left(\alpha\left(v_{1} \otimes v_{2}\right)\right)\right)=\gamma\left(v_{1} \otimes v_{2}\right.$, $\left.\lambda\left(w_{1} \otimes w_{2}\right)\right)=\gamma\left(v_{1} \otimes v_{2}, v_{1} \otimes v_{2}\right)=v_{1} \otimes v_{2}$ $\gamma\left(\lambda\left(\beta\left(v_{1} \otimes v_{2}\right)\right), v_{1} \otimes v_{2}\right)=\gamma\left(\lambda\left(w_{1} \otimes w_{2}\right), v_{1} \otimes v_{2}\right)$ $=\gamma\left(v_{1} \otimes v_{2}, v_{1} \otimes v_{2}\right)=\left(v_{1} \otimes v_{2}\right)$ , for all $v_{1} \otimes v_{2} \in \mathrm{T}_{1} \otimes \mathrm{T}_{2}$

(4) $\sigma: \mathrm{T}_{1} \otimes \mathrm{T}_{2} \rightarrow \mathrm{T}_{1} \otimes \mathrm{T}_{2}$

(a) $\alpha\left(\sigma\left(v_{1} \otimes v_{2}\right)\right)=\alpha\left(v_{1} \otimes v_{2}\right)=w_{1} \otimes w_{2}$, $\beta\left(v_{1} \otimes v_{2}\right)=w_{1} \otimes w_{2}$

We conclude $\alpha\left(\sigma\left(r_{1} \otimes v_{2}\right)\right)=\beta\left(v_{1} \otimes v_{2}\right)$, $\beta\left(\sigma\left(v_{1} \otimes v_{2}\right)\right)=\beta\left(v_{1} \otimes v_{2}\right)=w_{1} \otimes w_{2}, \alpha\left(v_{1} \otimes v_{2}\right)$ $=w_{1} \otimes w_{2}$

For any $v_{1} \otimes v_{2} \in \mathrm{T}_{1} \otimes \mathrm{T}_{2}$

(b) $\gamma\left(\sigma\left(v_{1} \otimes v_{2}\right), v_{1} \otimes v_{2}\right)=\gamma\left(v_{1} \otimes v_{2}, v_{1} \otimes v_{2}\right)$ $=v_{1} \otimes v_{2}, \lambda\left(\alpha\left(v_{1} \otimes v_{2}\right)\right)=\lambda\left(w_{1} \otimes w_{2}\right)=v_{1} \otimes v_{2}$ So we get $\gamma\left(\sigma\left(v_{1} \otimes v_{2}\right), v_{1} \otimes v_{2}\right)=\lambda\left(\alpha\left(v_{1} \otimes v_{2}\right)\right)$, And $\gamma\left(r_{1} \otimes v_{2}, \sigma\left(r_{1} \otimes v_{2}\right)\right)=\gamma\left(v_{1} \otimes v_{2}, r_{1} \otimes v_{2}\right)$ $=v_{1} \otimes v_{2}, \lambda\left(\beta\left(v_{1} \otimes v_{2}\right)\right)=\lambda\left(w_{1} \otimes w_{2}\right)=v_{1} \otimes v_{2}$

Hence we have $\gamma\left(v_{1} \otimes v_{2}, \sigma\left(v_{1} \otimes v_{2}\right)\right)$ $=\lambda\left(\beta\left(v_{1} \otimes v_{2}\right)\right)$ for all $v_{1} \otimes v_{2} \in \mathrm{T}_{1} \otimes \mathrm{T}_{2}$

We write $\sigma\left(v_{1} \otimes v_{2}\right)=\left(v_{1} \otimes v_{2}\right)^{-1}$ it refers to the invers element of $v_{1} \otimes v_{2} \in \mathrm{T}_{1} \otimes \mathrm{T}_{2}$ and $\lambda(\mathrm{x})=\tilde{x}$ is said to be the unite an item in $\mathrm{T}_{1} \otimes \mathrm{T}_{2}$ associaated to the element $\mathrm{x} \in \mathrm{Q}_{1} \otimes \mathrm{Q}_{2}$. Also we write $\gamma\left(v_{1} \otimes v_{2}, r_{1}^{\prime} \otimes v_{2}^{\prime}\right)=\left(v_{1} \otimes v_{2}\right)\left(v_{1}^{\prime} \otimes r_{2}^{\prime}\right), \mathrm{T}_{1} \otimes \mathrm{T}_{2}$ it refers to the groupoid and $\mathrm{Q}_{1} \otimes \mathrm{Q}_{2}$ is said to be the base

One more time, we say that $\mathrm{T}_{1} \otimes \mathrm{T}_{2}$ is a groupoid on $\mathrm{Q}_{1} \otimes \mathrm{Q}_{2}$.

\section{(1.8) Corollary:}

Let $\left(\mathrm{T}_{1}, \mathrm{Q}_{1}\right), \ldots,\left(\mathrm{T}_{n}, \mathrm{Q}_{n}\right)$ are groupoids then the Tensor product $\left(\otimes_{i=1}^{n} \mathrm{~T}_{i}, \otimes_{i=1}^{n} \mathrm{Q}_{i}\right)$ is a groupoid .

\section{Proof:}

Same the prove of theorem (1.7) .

\section{(1.9) Definition(8)}

Let $\mathrm{A}$ and $\mathrm{B}$ are both a subgroupoids of $\mathrm{T}$ (where $T$ be a groupoid ) then the direct sum $A \oplus B$ satisfy the following : (1) $\mathrm{A}+\mathrm{B}=\mathrm{T}$, (2) $\mathrm{A} \cap \mathrm{B}=\{0\}$.

\section{(1.7) Theorem:}

Let $\left(\mathrm{T}_{1}, \mathrm{Q}_{1}\right)$ and $\left(\mathrm{T}_{2}, \mathrm{Q}_{2}\right)$ are both groupoids then the direct sum $\left(\mathrm{T}_{1} \oplus \mathrm{T}_{2}, \mathrm{Q}_{1} \oplus \mathrm{Q}_{2}\right)$ be a groupoid .

\section{Proof:}

(1) $\alpha, \beta: T_{1} \oplus T_{2} \rightarrow Q_{1} \oplus Q_{2}$ $\alpha\left(v_{1} \oplus v_{2}\right)=w_{1} \oplus w_{2}, \beta\left(v_{1} \oplus v_{2}\right)=w_{1} \oplus w_{2}$, for all $v_{1} \oplus v_{2} \in \mathrm{T}_{1} \oplus \mathrm{T}_{2}$ and $w_{1} \oplus w_{2} \in \mathrm{Q}_{1} \oplus \mathrm{Q}_{2}$,

(2) $\lambda: Q_{1} \oplus Q_{2} \rightarrow \mathrm{T}_{1} \oplus \mathrm{T}_{2}$

$\lambda\left(w_{1} \oplus w_{2}\right)=v_{1} \oplus v_{2}$,

$(\alpha \mathrm{o} \lambda)\left(w_{1} \oplus w_{2}\right)=\alpha\left(\lambda\left(w_{1} \oplus w_{2}\right)\right)=\alpha\left(v_{1} \oplus v_{2}\right)=($ $\left.w_{1} \oplus w_{2}\right)$

$(\beta \mathrm{o} \lambda)\left(w_{1} \oplus w_{2}\right)=\beta\left(\lambda\left(w_{1} \oplus w_{2}\right)\right)=\beta\left(v_{1} \oplus v_{2}\right)=$ $\left(w_{1} \oplus w_{2}\right)$,

Where $\alpha \mathrm{o} \lambda=\mathrm{I}_{\mathrm{Q}_{1} \oplus \mathrm{Q}_{2}}, \beta \mathrm{o} \lambda=\mathrm{I}_{\mathrm{Q}_{1} \oplus \mathrm{Q}_{2}}$ and $\mathrm{I}_{\mathrm{Q}_{1} \oplus \mathrm{Q}_{2}}: \mathrm{Q}_{1} \oplus \mathrm{Q}_{2} \rightarrow \mathrm{Q}_{1} \oplus \mathrm{Q}_{2}$

(3) $\left(\mathrm{T}_{1} \oplus \mathrm{T}_{2}\right) *\left(\mathrm{~T}_{1} \oplus \mathrm{T}_{2}\right)=\left\{\left(v_{1} \oplus v_{2}, v_{1}^{\prime} \oplus v_{2}^{\prime}\right.\right.$ )$\in\left(\mathrm{T}_{1} \oplus \mathrm{T}_{2}\right) \times\left(\mathrm{T}_{1} \oplus \mathrm{T}_{2}\right)$ :

$\left.\alpha\left(v_{1} \oplus v_{2}\right)=\beta\left(v_{1}^{\prime} \oplus v_{2}^{\prime}\right)\right\}$ " fibre product of $\alpha$ and $\beta$ over $Q_{1} \oplus Q_{2}$ "

Such that

(a) $\gamma\left(v \oplus r, \gamma\left(v_{1} \oplus v_{1}, v_{2} \oplus v_{2}\right)\right)$

$=\gamma\left(v \oplus v, v_{2} \oplus v_{2}\right)=v_{2} \oplus v_{2}$

$\gamma\left(\gamma\left(r \oplus r, v_{1} \oplus v_{1}\right), v_{2} \oplus v_{2}\right)=\gamma\left(v_{1} \oplus v_{1}, v_{2} \oplus v_{2}\right)$

$=r_{2} \oplus r_{2}$

So we have $\gamma\left(v \oplus r, \gamma\left(v_{1} \oplus v_{1}, v_{2} \oplus v_{2}\right)\right)=$ $\gamma\left(\gamma\left(r \oplus v, v_{1} \oplus v_{1}\right), v_{2} \oplus v_{2}\right)$

For all $\left(v \oplus v, v_{1} \oplus v_{1}\right)$,

$\left(v_{1} \oplus v_{1}, v_{2} \oplus v_{2}\right) \in\left(\mathrm{T}_{1} \oplus \mathrm{T}_{2}\right) *\left(\mathrm{~T}_{1} \oplus \mathrm{T}_{2}\right)$

(b) $\left.\alpha\left(\gamma_{1} \oplus v_{1}, v_{2} \oplus v_{2}\right)\right)=\alpha\left(v_{2} \oplus v_{2}\right)=w_{2} \oplus w_{2}$

$\alpha\left(v_{2} \oplus v_{2}\right)=w_{2} \oplus w_{2}$

So we get $\alpha\left(\gamma\left(v_{1} \oplus v_{1}, v_{2} \oplus v_{2}\right)\right)=\alpha\left(v_{2} \oplus v_{2}\right)$,

$\beta\left(\gamma\left(v_{1} \oplus v_{1}, v_{2} \oplus v_{2}\right)\right)=\beta\left(v_{1} \oplus v_{1}\right)=w_{1} \oplus w_{1}$

$\beta\left(v_{1} \oplus v_{1}\right)=w_{1} \oplus w_{1}$

So we get , $\beta\left(\gamma\left(v_{1} \oplus v_{1}, v_{2} \oplus v_{2}\right)\right)=\beta\left(v_{1} \oplus v_{1}\right)$ for each $\left(v_{1} \oplus v_{1}, v_{2} \oplus v_{2}\right) \in\left(\mathrm{T}_{1} \oplus \mathrm{T}_{2}\right) *\left(\mathrm{~T}_{1} \oplus \mathrm{T}_{2}\right)$

(c) $\gamma\left(v_{1} \oplus v_{2}, \lambda\left(\alpha\left(v_{1} \oplus v_{2}\right)\right)\right)=\gamma\left(r_{1} \oplus v_{2}\right.$,

$\left.\lambda\left(w_{1} \oplus w_{2}\right)\right)=\gamma\left(v_{1} \oplus v_{2}, v_{1} \oplus v_{2}\right)=v_{1} \oplus v_{2}$

$\gamma\left(\lambda\left(\beta\left(v_{1} \oplus v_{2}\right)\right), v_{1} \oplus v_{2}\right)=\gamma\left(\lambda\left(w_{1} \oplus w_{2}\right), v_{1} \oplus v_{2}\right)$

$=\gamma\left(v_{1} \oplus v_{2}, v_{1} \oplus v_{2}\right)=\left(v_{1} \oplus v_{2}\right)$

, for all $v_{1} \oplus v_{2} \in \mathrm{T}_{1} \oplus \mathrm{T}_{2}$

(4) $\sigma: \mathrm{T}_{1} \oplus \mathrm{T}_{2} \rightarrow \mathrm{T}_{1} \oplus \mathrm{T}_{2}$

(a) $\alpha\left(\sigma\left(v_{1} \oplus v_{2}\right)\right)=\alpha\left(v_{1} \oplus v_{2}\right)=w_{1} \oplus w_{2}$,

$\beta\left(v_{1} \oplus v_{2}\right)=w_{1} \oplus w_{2}$

We conclude $\alpha\left(\sigma\left(v_{1} \oplus v_{2}\right)\right)=\beta\left(v_{1} \oplus v_{2}\right)$,

$\beta\left(\sigma\left(v_{1} \oplus v_{2}\right)\right)=\beta\left(v_{1} \oplus v_{2}\right)=w_{1} \oplus w_{2}, \alpha\left(v_{1} \oplus v_{2}\right)$

$=w_{1} \oplus w_{2}$

For any $v_{1} \oplus v_{2} \in \mathrm{T}_{1} \oplus \mathrm{T}_{2}$

(b) $\gamma\left(\sigma\left(v_{1} \oplus v_{2}\right), v_{1} \oplus v_{2}\right)=\gamma\left(v_{1} \oplus v_{2}, v_{1} \oplus v_{2}\right)$

$=v_{1} \oplus v_{2}, \lambda\left(\alpha\left(v_{1} \oplus v_{2}\right)\right)=\lambda\left(w_{1} \oplus w_{2}\right)=v_{1} \oplus v_{2}$ 
So we get $\gamma\left(\sigma\left(v_{1} \oplus v_{2}\right), v_{1} \oplus v_{2}\right)=\lambda\left(\alpha\left(v_{1} \oplus v_{2}\right)\right)$, And $\gamma\left(r_{1} \oplus v_{2}, \sigma\left(r_{1} \oplus v_{2}\right)\right)=\gamma\left(r_{1} \oplus v_{2}, v_{1} \oplus v_{2}\right)$ $=v_{1} \oplus v_{2}, \lambda\left(\beta\left(v_{1} \oplus v_{2}\right)\right)=\lambda\left(w_{1} \oplus w_{2}\right)=v_{1} \oplus v_{2}$ Hence we have $\gamma\left(v_{1} \oplus v_{2}, \sigma\left(v_{1} \oplus v_{2}\right)\right)$ $=\lambda\left(\beta\left(v_{1} \oplus v_{2}\right)\right)$ for all $v_{1} \oplus v_{2} \in \mathrm{T}_{1} \oplus \mathrm{T}_{2}$ We write $\sigma\left(v_{1} \oplus v_{2}\right)=\left(v_{1} \oplus v_{2}\right)^{-1}$ it refers to the invers element of $v_{1} \oplus v_{2} \in \mathrm{T}_{1} \oplus \mathrm{T}_{2}$ and $\lambda(\mathrm{x})=\tilde{x}$ is said to be the unite an item in $\mathrm{T}_{1} \oplus \mathrm{T}_{2}$ associaated to the element $\mathrm{x} \in \mathrm{Q}_{1} \oplus \mathrm{Q}_{2}$. Also we write $\gamma\left(v_{1} \oplus v_{2}, v_{1}^{\prime} \oplus v_{2}^{\prime}\right)=\left(v_{1} \oplus v_{2}\right)\left(v_{1}^{\prime} \oplus v_{2}^{\prime}\right), \mathrm{T}_{1} \oplus \mathrm{T}_{2}$ it refers to the groupoid and $\mathrm{Q}_{1} \oplus \mathrm{Q}_{2}$ is said to be the base

One more time, we say that $\mathrm{T}_{1} \oplus \mathrm{T}_{2}$ is a groupoid on $\mathrm{Q}_{1} \oplus \mathrm{Q}_{2}$.

\section{(1.11) Corollary:}

Let $\left(\mathrm{T}_{1}, \mathrm{Q}_{1}\right), \ldots,\left(\mathrm{T}_{n}, \mathrm{Q}_{n}\right)$ are groupoids then the Tensor product $\left(\oplus_{i=1}^{n} \mathrm{~T}_{i}, \oplus_{i=1}^{n} \mathrm{Q}_{i}\right)$ is a groupoid .

\section{Proof :}

Same way the proof of theorem (1.10) .

\section{(1.12)Definition(9)}

Let $\mathrm{T}_{1}$ and $\mathrm{T}_{2}$ are both a groupoids then the Cartesian Products of groupoids define by $\mathrm{T}_{1} \times \mathrm{T}_{2}=\left\{(\mathrm{a}, \mathrm{b}): \mathrm{a} \in \mathrm{T}_{1}, \mathrm{~b} \in \mathrm{T}_{2}\right\}$.

\section{(1.13) Theorem:}

Let $\left(\mathrm{T}_{1}, \mathrm{Q}_{1}\right)$ and $\left(\mathrm{T}_{2}, \mathrm{Q}_{2}\right)$ are both groupoids then the Cartesian Products $\left(T_{1} \times T_{2}, Q_{1} \times Q_{2}\right)$ be a groupoid .

\section{Proof:}

(1) $\alpha, \beta: T_{1} \times T_{2} \rightarrow Q_{1} \times Q_{2}$ $\alpha\left(v_{1}, v_{2}\right)=\left(w_{1}, w_{2}\right), \beta\left(v_{1}, v_{2}\right)=\left(w_{1}, w_{2}\right)$, for all $\left(v_{1}, v_{2}\right) \in \mathrm{T}_{1} \times \mathrm{T}_{2}$ and $\left(w_{1}, w_{2}\right) \in \mathrm{Q}_{1} \times \mathrm{Q}_{2}$,

(2) $\lambda: \mathrm{Q}_{1} \times \mathrm{Q}_{2} \rightarrow \mathrm{T}_{1} \times \mathrm{T}_{2}$

$\lambda\left(w_{1}, w_{2}\right)=\left(v_{1}, v_{2}\right)$,

$(\alpha \circ \lambda)\left(w_{1}, w_{2}\right)=\alpha\left(\lambda\left(w_{1}, w_{2}\right)\right)=\alpha\left(v_{1}, v_{2}\right)=$

$\left(w_{1}, w_{2}\right)$

$(\beta \circ \lambda)\left(w_{1}, w_{2}\right)=\beta\left(\lambda\left(w_{1}, w_{2}\right)\right)=\beta\left(v_{1}, v_{2}\right)=$ $\left(w_{1}, w_{2}\right)$,

Where $\alpha \mathrm{o} \lambda=\mathrm{I}_{\mathrm{Q}_{1} \times \mathrm{Q}_{2}}, \beta \mathrm{o} \lambda=\mathrm{I}_{\mathrm{Q}_{1} \times \mathrm{Q}_{2}}$ and $\mathrm{I}_{\mathrm{Q}_{1} \times \mathrm{Q}_{2}}$ : $\mathrm{Q}_{1} \times \mathrm{Q}_{2} \rightarrow \mathrm{Q}_{1} \times \mathrm{Q}_{2}$

(3) $\left(\mathrm{T}_{1} \times \mathrm{T}_{2}\right) *\left(\mathrm{~T}_{1} \times \mathrm{T}_{2}\right)=\left\{(v, v),\left(v^{\prime}, v^{\prime}\right)\right)$

$\in\left(\mathrm{T}_{1} \times \mathrm{T}_{2}\right) \times\left(\mathrm{T}_{1} \times \mathrm{T}_{2}\right):$

$\left.\alpha(v, r)=\beta\left(v^{\prime}, v^{\prime}\right)\right\}$ " fiber product of $\alpha$ and $\beta$ over $\mathrm{Q}_{1} \times \mathrm{Q}_{2}$ "
Such that

$$
\begin{aligned}
& \text { (a) } \gamma\left((v, r), \gamma\left(\left(v_{1}, v_{1}\right),\left(v_{2}, v_{2}\right)\right)\right)= \\
& \gamma\left((v, r),\left(v_{2}, v_{2}\right)\right)=\left(v_{2}, v_{2}\right) \\
& \gamma\left(\gamma\left((v, r),\left(v_{1}, v_{1}\right)\right),\left(v_{2}, v_{2}\right)\right)=\gamma\left(\left(v_{1}, v_{1}\right),\left(v_{2}, v_{2}\right)\right) \\
& \left.=v_{2}, v_{2}\right) \\
& \text { So we have } \gamma\left((v, r), \gamma\left(\left(v_{1}, v_{1}\right),\left(v_{2}, v_{2}\right)\right)\right)= \\
& \gamma\left(\gamma\left((r, v),\left(v_{1}, v_{1}\right)\right),\left(v_{2}, v_{2}\right)\right) \\
& \text { For all }\left((v, r),\left(v_{1}, v_{1}\right)\right),\left(\left(v_{1}, v_{1}\right),\left(v_{2}, r_{2}\right)\right) \\
& \in\left(\mathrm{T}_{1} \times \mathrm{T}_{2}\right) *\left(\mathrm{~T}_{1} \times \mathrm{T}_{2}\right) \\
& \text { (b) } \alpha\left(\gamma\left(\left(v_{1}, v_{1}\right),\left(v_{2}, r_{2}\right)\right)\right)=\alpha\left(v_{2}, v_{2}\right)=\left(w_{2}, w_{2}\right) \\
& \quad \alpha\left(v_{2}, v_{2}\right)=\left(w_{2}, w_{2}\right)
\end{aligned}
$$

So we get $\alpha\left(\gamma\left(\left(v_{1}, v_{1}\right),\left(v_{2}, v_{2}\right)\right)\right)=\alpha\left(v_{2}, v_{2}\right)$,

$\beta\left(\gamma\left(\left(v_{1}, v_{1}\right),\left(v_{2}, v_{2}\right)\right)\right)=\beta\left(v_{1}, v_{1}\right)=\left(w_{1}, w_{1}\right)$

$\beta\left(v_{1}, v_{1}\right)=\left(w_{1}, w_{1}\right)$

So we get , $\beta\left(\gamma\left(\left(v_{1}, v_{1}\right),\left(v_{2}, v_{2}\right)\right)\right)=\beta\left(v_{1}, v_{1}\right)$ for each $\left(\left(v_{1}, v_{1}\right),\left(v_{2}, v_{2}\right)\right) \in\left(\mathrm{T}_{1} \times \mathrm{T}_{2}\right) *\left(\mathrm{~T}_{1} \times \mathrm{T}_{2}\right)$

(c) $\gamma\left(\left(v_{1}, v_{2}\right), \lambda\left(\alpha\left(v_{1}, v_{2}\right)\right)\right)=\gamma\left(\left(v_{1}, v_{2}\right), \lambda\left(w_{1}, w_{2}\right)\right)$

$=\gamma\left(\left(v_{1}, v_{2}\right),\left(v_{1}, v_{2}\right)\right)=\left(v_{1}, v_{2}\right)$

$\gamma\left(\lambda\left(\beta\left(v_{1}, v_{2}\right)\right),\left(v_{1}, v_{2}\right)\right)=\gamma\left(\lambda\left(w_{1}, w_{2}\right),\left(v_{1}, v_{2}\right)\right)=$ $\gamma\left(\left(v_{1}, v_{2}\right),\left(v_{1}, v_{2}\right)\right)=\left(v_{1}, r_{2}\right)$

, for all $\left(v_{1}, r_{2}\right) \in \mathrm{T}_{1} \times \mathrm{T}_{2}$

(4) $\sigma: \mathrm{T}_{1} \times \mathrm{T}_{2} \rightarrow \mathrm{T}_{1} \times \mathrm{T}_{2}$

(a) $\alpha\left(\sigma\left(v_{1}, v_{2}\right)\right)=\alpha\left(v_{1}, v_{2}\right)=\left(w_{1}, w_{2}\right), \beta\left(v_{1}, v_{2}\right)=$ $\left(w_{1}, w_{2}\right)$

We conclude $\alpha\left(\sigma\left(v_{1}, v_{2}\right)\right)=\beta\left(v_{1}, v_{2}\right)$,

$\beta\left(\sigma\left(v_{1}, v_{2}\right)\right)=\beta\left(v_{1}, v_{2}\right)=\left(w_{1}, w_{2}\right), \alpha\left(v_{1}, v_{2}\right)=$ $\left(w_{1}, w_{2}\right)$

For any $\left(v_{1}, v_{2}\right) \in \mathrm{T}_{1} \times \mathrm{T}_{2}$

(b) $\gamma\left(\sigma\left(v_{1}, r_{2}\right),\left(v_{1}, v_{2}\right)\right)=\gamma\left(\left(v_{1}, v_{2}\right),\left(v_{1}, v_{2}\right)\right)=$ $\left(v_{1}, v_{2}\right), \lambda\left(\alpha\left(v_{1}, v_{2}\right)\right)=\lambda\left(w_{1}, w_{2}\right)=\left(v_{1}, v_{2}\right)$

So we get $\gamma\left(\sigma\left(v_{1}, v_{2}\right),\left(v_{1}, v_{2}\right)\right)=\lambda\left(\alpha\left(v_{1}, v_{2}\right)\right)$, And $\gamma\left(\left(v_{1}, v_{2}\right), \sigma\left(v_{1}, v_{2}\right)\right)=\gamma\left(\left(v_{1}, v_{2}\right),\left(v_{1}, v_{2}\right)\right)=$ $\left(v_{1}, v_{2}\right), \lambda\left(\beta\left(v_{1}, v_{2}\right)\right)=\lambda\left(w_{1}, w_{2}\right)=\left(v_{1}, v_{2}\right)$

Hence we have $\gamma\left(\left(v_{1}, v_{2}\right), \sigma\left(v_{1}, v_{2}\right)\right)=\lambda\left(\beta\left(v_{1}, v_{2}\right)\right)$ for all $\left(v_{1}, v_{2}\right) \in \mathrm{T}_{1} \times \mathrm{T}_{2}$

We write $\sigma\left(v_{1}, v_{2}\right)=\left(v_{1}, v_{2}\right)^{-1}$ it refers to the invers element of $\left(v_{1}, v_{2}\right) \in \mathrm{T}_{1} \times \mathrm{T}_{2}$ and $\lambda(\mathrm{x})=\tilde{x}$ is said to be the unite an item in $\mathrm{T}_{1} \times \mathrm{T}_{2}$ associaated to the element $x \in Q_{1} \times Q_{2}$. Also we write

$\gamma\left(\left(v_{1}, v_{2}\right),\left(v_{1}^{\prime}, r_{2}^{\prime}\right)\right)=\left(v_{1}, r_{2}\right)\left(v_{1}^{\prime}, v_{2}^{\prime}\right), \mathrm{T}_{1} \times \mathrm{T}_{2}$ it refers to the groupoid and $\mathrm{Q}_{1} \times \mathrm{Q}_{2}$ is said to be the base

One more time, we say that $\mathrm{T}_{1} \times \mathrm{T}_{2}$ is a groupoid on $Q_{1} \times Q_{2}$.

\section{(1.14) Corollary:}

Let $\left(\mathrm{T}_{1}, \mathrm{Q}_{1}\right), \ldots,\left(\mathrm{T}_{n}, \mathrm{Q}_{n}\right)$ are groupoids then the Cartisean product $\left(\times_{i=1}^{n} \mathrm{~T}_{i}, \times_{i=1}^{n} \mathrm{Q}_{i}\right)$ is a groupoid .

Proof: 
The prove same the way of proposition (1.13) .

\section{(1.15) Theorem}

Let $\left(\mathrm{T}_{1}, \mathrm{Q}_{1}\right)$ and $\left(\mathrm{T}_{2}, \mathrm{Q}_{2}\right)$ are both a groupoids then

(1) $\left(\left(\mathrm{T}_{1} \otimes \mathrm{T}_{2}\right) \oplus\left(\mathrm{T}_{1} \otimes \mathrm{T}_{2}\right),\left(\mathrm{Q}_{1} \otimes \mathrm{Q}_{2}\right) \oplus\left(\mathrm{Q}_{1} \otimes \mathrm{Q}_{2}\right)\right)$

(2) $\left(\left(\mathrm{T}_{1} \oplus \mathrm{T}_{2}\right) \otimes\left(\mathrm{T}_{1} \oplus \mathrm{T}_{2}\right),\left(\mathrm{Q}_{1} \oplus \mathrm{Q}_{2}\right) \otimes\left(\mathrm{Q}_{1} \oplus \mathrm{Q}_{2}\right)\right.$ are both groupoid

\section{Proof : of (1)}

(1) $\alpha, \beta:\left(\mathrm{T}_{1} \otimes \mathrm{T}_{2}\right) \oplus\left(\mathrm{T}_{1} \otimes \mathrm{T}_{2}\right) \rightarrow$ $\left(\mathrm{Q}_{1} \otimes \mathrm{Q}_{2}\right) \oplus\left(\mathrm{Q}_{1} \otimes \mathrm{Q}_{2}\right), \alpha\left(\left(v_{1} \otimes v_{2}\right) \oplus\left(v_{1} \otimes v_{2}\right)\right)=$ $\left(\left(w_{1} \otimes w_{2}\right) \oplus\left(w_{1} \otimes w_{2}\right)\right)$

$\beta\left(\left(v_{1} \otimes v_{2}\right) \oplus\left(v_{1} \otimes v_{2}\right)\right)=\left(\left(w_{1} \otimes w_{2}\right) \oplus\left(w_{1} \otimes w_{2}\right)\right)$

(2) $\lambda:\left(\mathrm{Q}_{1} \otimes \mathrm{Q}_{2}\right) \oplus\left(\mathrm{Q}_{1} \otimes \mathrm{Q}_{2}\right) \rightarrow\left(\mathrm{T}_{1} \otimes \mathrm{T}_{2}\right) \oplus\left(\mathrm{T}_{1} \otimes \mathrm{T}_{2}\right)$ ,$\lambda\left(\left(w_{1} \otimes w_{2}\right) \oplus\left(w_{1} \otimes w_{2}\right)\right)=$ $\left(\left(r_{1} \otimes v_{2}\right) \oplus\left(r_{1} \otimes v_{2}\right)\right)$

$(\alpha \mathrm{o} \lambda)\left(\left(w_{1} \otimes w_{2}\right) \oplus\left(w_{1} \otimes w_{2}\right)\right)=\alpha(\lambda(($ $\left.\left.w_{1} \otimes w_{2}\right) \oplus\left(w_{1} \otimes w_{2}\right)\right)=\alpha\left(\left(v_{1} \otimes v_{2}\right) \oplus\left(v_{1} \otimes v_{2}\right)\right)=$ $\left(\left(w_{1} \otimes w_{2}\right) \oplus\left(w_{1} \otimes w_{2}\right)\right)$

$(\beta \circ \lambda)\left(\left(w_{1} \otimes w_{2}\right) \oplus\left(w_{1} \otimes w_{2}\right)\right)=\beta(\lambda$

$\left.\left(\left(w_{1} \otimes w_{2}\right) \oplus\left(w_{1} \otimes w_{2}\right)\right)\right)=$

$\beta\left(\left(v_{1} \otimes v_{2}\right) \oplus\left(v_{1} \otimes v_{2}\right)\right)=\left(\left(w_{1} \otimes w_{2}\right) \oplus\left(w_{1} \otimes w_{2}\right)\right)$

So $\quad \alpha \mathrm{o} \lambda=\mathrm{I}_{\left(\mathrm{Q}_{1} \otimes \mathrm{Q}_{2}\right) \oplus\left(\mathrm{Q}_{1} \otimes \mathrm{Q}_{2}\right)}$ and $\beta \mathrm{o} \lambda=$ $\mathrm{I}_{\left(\mathrm{Q}_{1} \otimes \mathrm{Q}_{2}\right) \oplus\left(\mathrm{Q}_{1} \otimes \mathrm{Q}_{2}\right)}$

And

$\mathrm{I}_{\left(\mathrm{Q}_{1} \otimes \mathrm{Q}_{2}\right) \oplus\left(\mathrm{Q}_{1} \otimes \mathrm{Q}_{2}\right)}$ :

$\left(\mathrm{Q}_{1} \otimes \mathrm{Q}_{2}\right) \oplus\left(\mathrm{Q}_{1} \otimes \mathrm{Q}_{2}\right) \rightarrow\left(\mathrm{Q}_{1} \otimes \mathrm{Q}_{2}\right) \oplus\left(\mathrm{Q}_{1} \otimes \mathrm{Q}_{2}\right)$

(3) $\left(\mathrm{T}_{1} \otimes \mathrm{T}_{2}\right) \oplus\left(\mathrm{T}_{1} \otimes \mathrm{T}_{2}\right) *\left(\mathrm{~T}_{1} \otimes \mathrm{T}_{2}\right) \oplus\left(\mathrm{T}_{1} \otimes \mathrm{T}_{2}\right)=$ $\left\{\left(\left(\left(v_{1} \otimes v_{2}\right) \oplus\left(v_{1} \otimes v_{2}\right)\right),\left(\left(v_{1}^{\prime} \otimes v_{2}^{\prime}\right) \oplus\left(r_{1}^{\prime} \otimes\right.\right.\right.\right.$

$\left.\left.\left.r_{2}^{\prime}\right)\right)\right) \in$

$\left(\left(\mathrm{T}_{1} \otimes \mathrm{T}_{2}\right) \oplus\left(\mathrm{T}_{1} \otimes \mathrm{T}_{2}\right)\right) \times\left(\left(\mathrm{T}_{1} \otimes \mathrm{T}_{2}\right) \oplus\left(\mathrm{T}_{1} \otimes \mathrm{T}_{2}\right)\right):$ $\left.\alpha\left(\left(v_{1} \otimes v_{2}\right) \oplus\left(v_{1} \otimes v_{2}\right)\right)=\beta\left(\left(v_{1}^{\prime} \otimes v_{2}^{\prime}\right) \oplus\left(v_{1}^{\prime} \otimes v_{2}^{\prime}\right)\right)\right\}$

" fibre product of $\alpha$ and $\beta$ over $\left(\left(Q_{1} \otimes Q_{2}\right) \oplus\left(Q_{1} \otimes Q_{2}\right)\right)$ "such that

(a) $\quad \gamma\left(((v \otimes r) \oplus(v \otimes r)), \gamma\left(\left(\left(v_{1} \otimes v_{2}\right) \oplus\left(v_{1} \otimes v_{2}\right)\right)\right.\right.$, $\left.\left.\left(\left(v_{1}^{\prime} \otimes v_{2}^{\prime}\right) \oplus\left(v_{1}^{\prime} \otimes v_{2}^{\prime}\right)\right)\right)\right)=$

$\gamma\left(((v \otimes r) \oplus(v \otimes r)),\left(\left(v_{1}^{\prime} \otimes v_{2}^{\prime}\right) \oplus\left(v_{1}^{\prime} \otimes v_{2}^{\prime}\right)\right)\right) \quad=$ $\left(\left(v_{1}^{\prime} \otimes v_{2}^{\prime}\right) \oplus\left(r_{1}^{\prime} \otimes r_{2}^{\prime}\right)\right)$

And

$\gamma\left(\gamma\left(((r \otimes v) \oplus(r \otimes r)),\left(\left(v_{1} \otimes v_{2}\right) \oplus\left(r_{1} \otimes v_{2}\right)\right)\right),\left(\left(\left(v_{1}^{\prime}\right.\right.\right.\right.$

$\left.\left.\left.\otimes v_{2}^{\prime}\right) \oplus\left(r_{1}^{\prime} \otimes v_{2}^{\prime}\right)\right)\right)=$ $\gamma\left(\left(\left(v_{1} \otimes v_{2}\right) \oplus\left(r_{1} \otimes v_{2}\right)\right),\left(\left(v_{1}^{\prime} \otimes v_{2}^{\prime}\right) \oplus\left(v_{1}^{\prime} \otimes v_{2}^{\prime}\right)\right)\right)=$ $\left(\left(v_{1}^{\prime} \otimes r_{2}^{\prime}\right) \oplus\left(v_{1}^{\prime} \otimes v_{2}^{\prime}\right)\right)$

So we get

$\gamma\left(((v \otimes r) \oplus(v \otimes r)), \gamma\left(\left(\left(v_{1} \otimes v_{2}\right) \oplus\left(v_{1} \otimes v_{2}\right)\right)\right.\right.$,

$\left.\left.\left(\left(v_{1}^{\prime} \otimes r_{2}^{\prime}\right) \oplus\left(r_{1}^{\prime} \otimes r_{2}^{\prime}\right)\right)\right)\right)=$

$\gamma\left(\gamma\left(((r \otimes r) \oplus(r \otimes r)),\left(\left(v_{1} \otimes v_{2}\right) \oplus\left(r_{1} \otimes v_{2}\right)\right)\right),\left(\left(\left(v_{1}^{\prime}\right.\right.\right.\right.$

$\left.\left.\left.\otimes v_{2}^{\prime}\right) \oplus\left(r_{1}^{\prime} \otimes v_{2}^{\prime}\right)\right)\right)$

For all $((r \otimes r) \oplus(r \otimes r)),\left(\left(r_{1} \otimes v_{2}\right) \oplus\left(v_{1} \otimes v_{2}\right)\right)$, $\left(\left(\left(v_{1} \otimes v_{2}\right) \oplus\left(r_{1} \otimes v_{2}\right)\right),\left(\left(v_{1}^{\prime} \otimes v_{2}^{\prime}\right) \oplus\left(r_{1}^{\prime} \otimes v_{2}^{\prime}\right)\right)\right)$

$\in\left(\mathrm{T}_{1} \otimes \mathrm{T}_{2} \oplus \mathrm{T}_{1} \otimes \mathrm{T}_{2}\right) *\left(\mathrm{~T}_{1} \otimes \mathrm{T}_{2} \oplus \mathrm{T}_{1} \otimes \mathrm{T}_{2}\right)$

(b)

$\alpha\left(\gamma\left(\left(\left(v_{1} \otimes v_{2}\right) \oplus\left(v_{1} \otimes v_{2}\right)\right),\left(\left(r_{1}^{\prime} \otimes v_{2}^{\prime}\right) \oplus\left(v_{1}^{\prime} \otimes v_{2}^{\prime}\right)\right.\right.\right.$

)$)=\alpha\left(\left(v_{1}^{\prime} \otimes r_{2}^{\prime}\right) \oplus\left(v_{1}^{\prime} \otimes v_{2}^{\prime}\right)\right)$

$=\left(\left(w_{1}^{\prime} \otimes w_{2}^{\prime}\right) \oplus\left(w_{1}^{\prime} \otimes w_{2}^{\prime}\right)\right)$

And $\alpha\left(\left(v_{1}^{\prime} \otimes v_{2}^{\prime}\right) \oplus\left(v_{1}^{\prime} \otimes v_{2}^{\prime}\right)\right)$

$=\left(\left(w_{1}^{\prime} \otimes w_{2}^{\prime}\right) \oplus\left(w_{1}^{\prime} \otimes w_{2}^{\prime}\right)\right)$

So we get

$\alpha\left(\gamma\left(\left(\left(v_{1} \otimes v_{2}\right) \oplus\left(r_{1} \otimes v_{2}\right)\right),\left(\left(v_{1}^{\prime} \otimes v_{2}^{\prime}\right) \oplus\left(v_{1}^{\prime} \otimes v_{2}^{\prime}\right)\right)\right.\right.$

)$)=\alpha\left(\left(v_{1}^{\prime} \otimes r_{2}^{\prime}\right) \oplus\left(r_{1}^{\prime} \otimes r_{2}^{\prime}\right)\right)$

$\beta\left(\gamma\left(\left(\left(v_{1} \otimes v_{2}\right) \oplus\left(v_{1} \otimes v_{2}\right)\right),\left(\left(v_{1}^{\prime} \otimes v_{2}^{\prime}\right) \oplus\left(v_{1}^{\prime} \otimes v_{2}^{\prime}\right)\right)\right.\right.$

)$)=\beta\left(\left(v_{1} \otimes r_{2}\right) \oplus\left(r_{1} \otimes r_{2}\right)\right)=$

$\left(\left(w_{1} \otimes w_{2}\right) \oplus\left(w_{1} \otimes w_{2}\right)\right)$

$\beta\left(\left(v_{1} \otimes v_{2}\right) \oplus\left(v_{1} \otimes v_{2}\right)\right)=\left(\left(w_{1} \otimes w_{2}\right) \oplus\left(w_{1} \otimes w_{2}\right)\right)$

So we get

$\beta\left(\gamma\left(\left(\left(v_{1} \otimes v_{2}\right) \oplus\left(v_{1} \otimes v_{2}\right)\right),\left(\left(v_{1}^{\prime} \otimes v_{2}^{\prime}\right) \oplus\left(v_{1}^{\prime} \otimes v_{2}^{\prime}\right)\right)\right.\right.$ )$)=\beta\left(\left(v_{1} \otimes v_{2}\right) \oplus\left(r_{1} \otimes v_{2}\right)\right)$

For all

$\left(\left(\left(v_{1} \otimes v_{2}\right) \oplus\left(r_{1} \otimes v_{2}\right)\right),\left(\left(v_{1}^{\prime} \otimes v_{2}^{\prime}\right) \oplus\left(v_{1}^{\prime} \otimes v_{2}^{\prime}\right)\right)\right) \in$ $\left(\mathrm{T}_{1} \otimes \mathrm{T}_{2} \oplus \mathrm{T}_{1} \otimes \mathrm{T}_{2}\right) *\left(\mathrm{~T}_{1} \otimes \mathrm{T}_{2} \oplus \mathrm{T}_{1} \otimes \mathrm{T}_{2}\right)$

(c) $\gamma\left(\left(\left(v_{1} \otimes v_{2}\right) \oplus\left(v_{1} \otimes v_{2}\right)\right)\right.$, $\left.\lambda\left(\alpha\left(\left(v_{1} \otimes r_{2}\right) \oplus\left(r_{1} \otimes v_{2}\right)\right)\right)\right)=$

$\gamma\left(\left(\left(v_{1} \otimes v_{2}\right) \oplus\left(r_{1} \otimes v_{2}\right)\right)\right.$, $\left.\lambda\left(\left(w_{1} \otimes w_{2}\right) \oplus\left(w_{1} \otimes w_{2}\right)\right)\right)=$ $\gamma\left(\left(\left(v_{1} \otimes v_{2}\right) \oplus\left(v_{1} \otimes v_{2}\right)\right),\left(\left(v_{1} \otimes v_{2}\right) \oplus\left(r_{1} \otimes v_{2}\right)\right)\right)$

$=\left(\left(v_{1} \otimes v_{2}\right) \oplus\left(v_{1} \otimes v_{2}\right)\right)$ 


$$
\begin{aligned}
& \gamma\left(\lambda\left(\beta\left(\left(v_{1} \otimes v_{2}\right) \oplus\left(v_{1} \otimes v_{2}\right)\right)\right),\right. \\
& \left.\left(\left(r_{1} \otimes v_{2}\right) \oplus\left(v_{1} \otimes v_{2}\right)\right)\right)= \\
& \gamma\left(\lambda\left(\left(w_{1} \otimes w_{2}\right) \oplus\left(w_{1} \otimes w_{2}\right)\right),\left(\left(v_{1} \otimes v_{2}\right) \oplus\left(v_{1} \otimes v_{2}\right)\right)\right) \\
& =\gamma\left(\left(\left(v_{1} \otimes v_{2}\right) \oplus\left(v_{1} \otimes v_{2}\right)\right),\left(\left(r_{1} \otimes r_{2}\right) \oplus\left(v_{1} \otimes v_{2}\right)\right)\right)= \\
& \left(\left(r_{1} \otimes v_{2}\right) \oplus\left(v_{1} \otimes v_{2}\right)\right)
\end{aligned}
$$

For all $\left(\left(v_{1} \otimes v_{2}\right) \oplus\left(v_{1} \otimes v_{2}\right)\right) \in\left(\mathrm{T}_{1} \otimes \mathrm{T}_{2} \oplus \mathrm{T}_{1} \otimes \mathrm{T}_{2}\right)$

(4) $\sigma:\left(\mathrm{T}_{1} \otimes \mathrm{T}_{2}\right) \oplus\left(\mathrm{T}_{1} \otimes \mathrm{T}_{2}\right) \rightarrow\left(\mathrm{T}_{1} \otimes \mathrm{T}_{2}\right) \oplus\left(\mathrm{T}_{1} \otimes \mathrm{T}_{2}\right)$

(a) $\alpha\left(\sigma\left(\left(v_{1} \otimes v_{2}\right) \oplus\left(v_{1} \otimes v_{2}\right)\right)\right)$

$=\alpha\left(v_{1} \otimes v_{2}\right) \oplus\left(v_{1} \otimes v_{2}\right)=\left(\left(w_{1} \otimes w_{2}\right) \oplus\left(w_{1} \otimes w_{2}\right)\right)$

$\beta\left(\left(v_{1} \otimes v_{2}\right) \oplus\left(v_{1} \otimes v_{2}\right)\right)=\left(\left(w_{1} \otimes w_{2}\right) \oplus\left(w_{1} \otimes w_{2}\right)\right)$

So we have $\alpha\left(\sigma\left(\left(v_{1} \otimes v_{2}\right) \oplus\left(v_{1} \otimes v_{2}\right)\right)\right)=$

$\beta\left(\left(v_{1} \otimes v_{2}\right) \oplus\left(v_{1} \otimes v_{2}\right)\right)$ for any

$\left(\left(v_{1} \otimes v_{2}\right) \oplus\left(v_{1} \otimes v_{2}\right)\right) \in\left(\mathrm{T}_{1} \otimes \mathrm{T}_{2} \oplus \mathrm{T}_{1} \otimes \mathrm{T}_{2}\right)$

(b) $\gamma\left(\sigma\left(\left(v_{1} \otimes v_{2}\right) \oplus\left(v_{1} \otimes v_{2}\right)\right)\right)$,

$\left.\left(\left(r_{1} \otimes r_{2}\right) \oplus\left(v_{1} \otimes v_{2}\right)\right)\right)=$

$\left.\gamma\left(\left(v_{1} \otimes v_{2}\right) \oplus\left(v_{1} \otimes v_{2}\right)\right),\left(\left(v_{1} \otimes v_{2}\right) \oplus\left(v_{1} \otimes v_{2}\right)\right)\right)$

$=\left(\left(v_{1} \otimes v_{2}\right) \oplus\left(v_{1} \otimes v_{2}\right)\right)$

$\lambda\left(\alpha\left(\left(v_{1} \otimes v_{2}\right) \oplus\left(v_{1} \otimes v_{2}\right)\right)\right)=$

$\lambda\left(\left(w_{1} \otimes w_{2}\right) \oplus\left(w_{1} \otimes w_{2}\right)\right)=\left(\left(v_{1} \otimes v_{2}\right) \oplus\left(v_{1} \otimes v_{2}\right)\right)$

So we get $\gamma\left(\sigma\left(\left(\left(v_{1} \otimes v_{2}\right) \oplus\left(v_{1} \otimes v_{2}\right)\right)\right.\right.$

),$\left.\left(\left(v_{1} \otimes v_{2}\right) \oplus\left(v_{1} \otimes v_{2}\right)\right)\right)=$

$\lambda\left(\alpha\left(\left(v_{1} \otimes v_{2}\right) \oplus\left(v_{1} \otimes v_{2}\right)\right)\right)$

And

$\left.\gamma\left(\sigma\left(\left(v_{1} \otimes v_{2}\right) \oplus\left(v_{1} \otimes v_{2}\right)\right)\right),\left(\left(r_{1} \otimes v_{2}\right) \oplus\left(v_{1} \otimes v_{2}\right)\right)\right)$

$=\gamma\left(\left(\left(v_{1} \otimes v_{2}\right) \oplus\left(v_{1} \otimes v_{2}\right)\right),\left(\left(v_{1} \otimes v_{2}\right) \oplus\left(v_{1} \otimes v_{2}\right)\right)\right)$

$=\left(\left(v_{1} \otimes v_{2}\right) \oplus\left(v_{1} \otimes v_{2}\right)\right)$

$\lambda\left(\beta\left(\left(v_{1} \otimes v_{2}\right) \oplus\left(v_{1} \otimes v_{2}\right)\right)\right)=$

$\lambda\left(w_{1} \otimes w_{2}\right) \oplus\left(w_{1} \otimes w_{2}\right)=\left(\left(v_{1} \otimes v_{2}\right) \oplus\left(v_{1} \otimes v_{2}\right)\right)$

So we get

$\left.\gamma\left(\sigma\left(\left(v_{1} \otimes v_{2}\right) \oplus\left(v_{1} \otimes v_{2}\right)\right)\right),\left(\left(v_{1} \otimes v_{2}\right) \oplus\left(v_{1} \otimes v_{2}\right)\right)\right)=$ $\lambda\left(\beta\left(\left(r_{1} \otimes r_{2}\right) \oplus\left(r_{1} \otimes v_{2}\right)\right)\right)$

For all $\left(\left(v_{1} \otimes v_{2}\right) \oplus\left(v_{1} \otimes v_{2}\right)\right) \in\left(\mathrm{T}_{1} \otimes \mathrm{T}_{2} \oplus \mathrm{T}_{1} \otimes \mathrm{T}_{2}\right)$ $\sigma\left(\left(v_{1} \otimes v_{2}\right) \oplus\left(v_{1} \otimes v_{2}\right)\right)=\left(\left(v_{1} \otimes v_{2}\right) \oplus\left(v_{1} \otimes v_{2}\right)\right)^{-1}$

called the inverse element of $\left(\left(v_{1} \otimes v_{2}\right) \oplus\left(v_{1} \otimes v_{2}\right)\right)$

$\in\left(\mathrm{T}_{1} \otimes \mathrm{T}_{2} \oplus \mathrm{T}_{1} \otimes \mathrm{T}_{2}\right) \quad$ and $\lambda(\mathrm{x})=\tilde{x}$ refers to the unite element in $\left(\mathrm{T}_{1} \otimes \mathrm{T}_{2}\right) \oplus\left(\mathrm{T}_{1} \otimes \mathrm{T}_{2}\right)$

associated to the element

$\mathrm{x} \in\left(\left(\mathrm{Q}_{1} \otimes \mathrm{Q}_{2}\right) \oplus\left(\mathrm{Q}_{1} \otimes \mathrm{Q}_{2}\right)\right)$.

Also we rewrite

$\gamma\left(\left(\left(v_{1} \otimes v_{2}\right) \oplus\left(v_{1} \otimes v_{2}\right)\right),\left(\left(v_{1}^{\prime} \otimes v_{2}^{\prime}\right) \oplus\left(v_{1}^{\prime} \otimes v_{2}^{\prime}\right)\right)\right)$

$\left.=\left(v_{1} \otimes v_{2}\right) \oplus\left(v_{1} \otimes v_{2}\right)\right)\left(\left(v_{1}^{\prime} \otimes v_{2}^{\prime}\right) \oplus\left(v_{1}^{\prime} \otimes v_{2}^{\prime}\right)\right)$.

$\left(\mathrm{T}_{1} \otimes \mathrm{T}_{2} \oplus \mathrm{T}_{1} \otimes \mathrm{T}_{2}\right)$ it refers to the groupoid and $\left(\mathrm{Q}_{1} \otimes \mathrm{Q}_{2}\right) \oplus\left(\mathrm{Q}_{1} \otimes \mathrm{Q}_{2}\right)$ is called base .

Also we say that $\left(\mathrm{T}_{1} \otimes \mathrm{T}_{2} \oplus \mathrm{T}_{1} \otimes \mathrm{T}_{2}\right)$ is a groupoid on $\left(\left(Q_{1} \otimes Q_{2}\right) \oplus\left(Q_{1} \otimes Q_{2}\right)\right)$.

The prove of (2) same the prove of (1) .

2.

Subgroupoid, normal subgroupoid, morphism of groupoid, Krenal .

\section{(2.1) Definition(5)}

"Let (T,Q) be any groupoid .A subgroupoid of $(T, Q)$ is a pair $(H, A)$ of sub sets, $\mathrm{H} \subseteq \mathrm{T}$ and $\mathrm{A} \subseteq \mathrm{Q}$ with

$\alpha(\mathrm{H}) \subseteq \mathrm{A}, \beta(\mathrm{H}) \subseteq \mathrm{A}, \lambda(\mathrm{A}) \subseteq \mathrm{H}$ and $\mathrm{H}$ is closed under partial composition $\gamma$ and inversion $\sigma$ of T . In other

word $\mathrm{H}$ is a groupoid of base A such that"

(i) $\alpha_{\mathrm{H}}=\left.\alpha\right|_{\mathrm{H}}, \beta_{\mathrm{H}}=\left.\beta\right|_{\mathrm{H}}$ and $\lambda_{\mathrm{A}}=\left.\lambda\right|_{\mathrm{A}}$.

(ii) $\gamma_{\mathrm{H}}=\left.\gamma\right|_{\mathrm{H} * \mathrm{H}} ; \sigma_{\mathrm{H}}=\left.\sigma\right|_{\mathrm{H}}$.

and a subgroupoid $(\mathrm{H}, \mathrm{A})$ of a groupoid $(\mathrm{T}, \mathrm{Q})$ is called wide if $A=Q$.

(2.2) Example(5)

Let $(T, Q)$ be any groupoid then a set $\widetilde{Q}=\{\tilde{x}$ $: \mathrm{X} \in \mathrm{Q}\}=\lambda(\mathrm{Q})$ is a wide subgroupoid of $\mathrm{T}$ that is called the 
base subgroupoid of $(T, Q)$.

\section{(2.3) Definition(3)}

Let (T,Q) be any groupoid . A normal subgroupoid of $(T, Q)$ is a wide subgroupoid $H$ such that for

all $\mathrm{h} \in \mathrm{H}$ and $\mathrm{t} \in \mathrm{T}$ with $\alpha(\mathrm{t})=\alpha(\mathrm{h})=\beta(\mathrm{h})$ we have tht ${ }^{-1} \in \mathrm{H}$.

\section{(2.4) Definition(7)}

"A morphism of groupoid is a pair of maps $\left(\mathrm{f}, \mathrm{f}_{0}\right):(\mathrm{T}, \mathrm{Q}) \rightarrow\left(\mathrm{T}^{\prime}, \mathrm{Q}^{\prime}\right) \quad$ such that $\quad \alpha^{\prime} \mathrm{of}=\mathrm{f}_{0} \mathrm{O} \alpha$ ,$\beta^{\prime}$ of $=\mathrm{f}_{0} \mathrm{o} \beta$ and $\mathrm{f}\left(\gamma\left(\mathrm{t}, \mathrm{t}^{\prime}\right)\right)=\gamma^{\prime}\left(\mathrm{f}(\mathrm{t}), \mathrm{f}\left(\mathrm{t}^{\prime}\right)\right)$ for all $\left(\mathrm{t}, \mathrm{t}^{\prime}\right) \in \mathrm{T} * \mathrm{~T}^{\prime \prime}$. An isomorphism of groupoids is a morphism of groupoids such that

$\mathrm{f}: \mathrm{T} \rightarrow \mathrm{T}^{\prime}$ is bijeective map

\section{(2.5) Proposition}

Let $f^{\prime}:\left(f, f_{0}\right):\left(\mathrm{T}_{1}, \mathrm{Q}_{1}\right) \rightarrow\left(\mathrm{T}_{2}, \mathrm{Q}_{2}\right)$ be a morrphism and Let $g^{\prime}:\left(g, g_{0}\right):\left(\mathrm{T}_{2}, \mathrm{Q}_{2}\right) \rightarrow\left(\mathrm{T}_{3}, \mathrm{Q}_{3}\right)$ be a morphim

then $g^{\prime}$ of $f^{\prime}:\left(\mathrm{T}_{1}, \mathrm{Q}_{1}\right) \rightarrow\left(\mathrm{T}_{3}, \mathrm{Q}_{3}\right)$ is a morphiism .

Proof:

Since $\mathrm{f}^{\prime}$ and $g^{\prime}$ are both a morphism then

$\alpha^{\prime}$ of $=f_{0}$ o $\alpha$ and $\beta^{\prime}$ of $=f_{0} o \beta$, and

$\mathrm{f}_{0} \mathrm{o} \alpha=\beta^{\prime}$ of , then $\alpha^{\prime}$ of $=\mathrm{f}_{0} \mathrm{o} \beta$

so $g$ 'of' it's a morphism .

\section{(2.6)corollary}

Let $g:\left(f_{1}, f_{1}^{\prime}\right):\left(\mathrm{T}_{1}, \mathrm{Q}_{1}\right) \rightarrow\left(\mathrm{T}_{2}, \mathrm{Q}_{2}\right)$ and $g_{1}$ : $\left(\mathrm{T}_{2}, \mathrm{Q}_{2}\right) \rightarrow\left(\mathrm{T}_{3}, \mathrm{Q}_{3}\right) \ldots . g_{n}:\left(\mathrm{T}_{\mathrm{n}-1}, \mathrm{Q}_{\mathrm{n}-1}\right) \rightarrow\left(\mathrm{T}_{\mathrm{n}}, \mathrm{Q}_{\mathrm{n}}\right)$ are a morphism

then the composition $g_{n} \circ g_{n-1} \mathrm{o} \ldots . \mathrm{og}: \mathrm{S} \rightarrow \mathrm{S}_{\mathrm{n}}$ be a morphism .

\section{Proof}

The prove same the way of proposition (2.5) .

\section{(2.7)Definition(6)}

"Let $\left(\mathrm{f}, \mathrm{f}_{0}\right):(\mathrm{T}, \mathrm{Q}) \rightarrow\left(\mathrm{T}^{\prime}, \mathrm{Q}^{\prime}\right)$ be a morphism of groupoids then

(i) The kernal of $f$ is the set kerf $=\{t \in T$

|f(t) $\left.\in \lambda^{\prime}\left(Q^{\prime}\right)\right\}$.

(ii) If $\mathrm{Q}^{\prime} \mathrm{Q}^{\prime}$ and $\mathrm{f}_{0}=\mathrm{I}_{\mathrm{Q}}$ then $\mathrm{f}$ is called a morphism over Q".

\section{(2.8) Remark(6)}

If $\left(f, f_{0}\right):(T, Q) \rightarrow\left(T^{\prime}, Q^{\prime}\right)$ be a morphism of groupoids then the kerf is normal subgroupoid of T but $\mathrm{f}(\mathrm{T})$

is not necessarily subgroupoid of $\mathrm{T}^{\prime}$ since whenever $\gamma^{\prime}\left(\mathrm{f}(\mathrm{t}), \mathrm{f}\left(\mathrm{t}^{\prime}\right)\right)$ is defined in $\mathrm{T}^{\prime}, \gamma\left(\mathrm{t}, \mathrm{t}^{\prime}\right)$ is not necessarily

the base map $\mathrm{f}_{0}: \mathrm{Q} \rightarrow \mathrm{Q}^{\prime}$ is injective .Also $\left(\mathrm{f}, \mathrm{f}_{0}\right)$ induces a homomorphism of group say ${ }_{x} f_{x}:{ }_{x} T_{x}$ $\rightarrow_{\mathrm{fo}(\mathrm{x})} \mathrm{T}_{\mathrm{fO}(\mathrm{x})}^{\prime}$,

for all $\mathrm{x} \in \mathrm{T}$.

\section{(2.9) Definition(4)}

Let $(T, Q)$ be any groupoid, we say that $T$ is transitive groupoid if transitor $\tau: \mathrm{T} \rightarrow \mathrm{Q} \times \mathrm{Q}$ ,$\tau(\mathrm{t})=(\beta(\mathrm{t}), \alpha(\mathrm{t}))$ is

surjective map i.e. agroupoid is transitive if any two points of its base can be joined by an element of

the groupoid .

\section{(2.10) Example(4)}

Any trivial groupoid its transitive groupoid

\section{Solution:}

Let $\eta$ be a group and $\mathrm{T}=\mathrm{Q} \times \mathfrak{\eta} \times \mathrm{Q}$ is a groupoid

$\alpha, \beta: \mathrm{Q} \times \dot{n} \times \mathrm{Q} \rightarrow \mathrm{Q}$

$\alpha(q, x, q)=q, \beta(q, x, q)=q$

$\tau(\mathrm{q}, \mathrm{x}, \mathrm{q})=(\mathrm{q}, \mathrm{q})$

we have $\tau(q, x, q)=(\beta(q), \alpha(q))$

So any trivial agroupoid its transitive groupoid . 


\section{References:}

(1) Allen P.J. , Kim H.S. , Neggers J. , "Sevral types of groupoids induced by two variable functions", springer 2016 .

(2) Brown, R., "Topology and Groupoids ”,Deganwy,United Kingdom (2014).

(3) Du C.Y., Chen B.,Wang R.A., "Groupoid of morphism of Groupoids" ,Cambridge, USA, 2017

(4) Jesus A. , Martin C. , "On Groupoid Gardings" ,Journal of Geometry and Physics Vol.123,Iss.3, ,P.P.61-70, January 2018.

(5) Majeed .T.H. , "On Some Results of Topological Groupoid”, (IHSCICONF 2017) IOP Conf.seeries: Journal of Physics, 1003, P.P.1-8, 2018.

(6) Myrnouri H., "Dual Groupoid of an Abelian Groupoid",21 th seminar an Mathematical Analysis and it's Application 26-27 November, Islamic Azad University, Hamedan , Iran (2015) .

(7) Ng H.K. , "The Fundamental Groupoid", The University of Adelaidr AMSI,(2014) .

(8) Ortiz C. ,Hoyo M.D,"Morita Equivalence Vector bundles", Journal of pure Applied Algebra,Vol.214, Iss. 6,p.p. 750-768, 2016.

(9) Pledger. K.H. , "Internal Direct Products of Groupoids", Journal of Algebra, Vol.217 , P.P.599-627, Iss. 2,1999. 


\author{
أنواع معينه من الفئات الزمروية

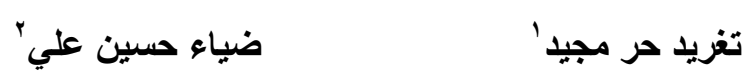 \\ قسم الرياضيات / كلية التربية / الجامعة المستنصرية \\ taghreedmajeed@yahoo.com $^{1}$ \\ deyaa h_ali@yahoo.com ${ }^{2}$
}

(المستخلص:

نحاول الاهتمام في هذا البحث و النظر لأهميته في الحصول على بعض النتائج و النظريات و نسعى لإعطاء بعض الأمثلة و توضيحها للفئه الزمرويه .

الكلمات المفتاحية:

الفئات الزمروية ، فئة دسكاريتس الزمروية ، الجمع المباشر لفئتن زمروية ، الضرب التسوري لفنتين زمروية ، فعل الفئة الزمروية . 